\title{
Epidemiology of Chlamydia trachomatis in the Middle East and North Africa: Updated
}

systematic review, meta-analyses, and meta-regressions

Alex Smolak, ${ }^{1}$ Hiam Chemaitelly, ${ }^{1}$ and Laith J Abu-Raddad ${ }^{1,2^{*}}$

${ }^{1}$ Infectious Disease Epidemiology Group, Weill Cornell Medicine-Qatar, Cornell University, Doha, Qatar

2Department of Healthcare Policy and Research, Weill Cornell Medicine, Cornell University, New York, USA

\section{BACKGROUND}

- Chlamydia trachomatis (CT) epidemiology in the Middle East and North Africa (MENA), the region with the highest rates of primary infertility, is poorly understood.

- The World Health Organization (WHO) recently formulated the 'Global Health Sector Strategy on STIs, 2016-2021' with the aim of ending STI epidemics as a public health concern by 2030.

OBJECTIVE

- To inform efforts attending to the Global Health Sector Strategy on STIs 2016-2012 through a comprehensive epidemiological assessment of CT infection in MENA.

\section{METHODOLOGY}

- Systematic review of all epidemiological evidence for CT infection in MENA up to November 2017, and reporting findings following the PRISMA guidelines (Fig. 1).

- Data synthesized by at-risk population and type of assay used for CT infection ascertainment.

- Pooled mean CT prevalence in at-risk populations and by type of assay used for CT infection ascertainment estimated using random-effects meta-analyses with inverse variance weighting (Table 1).

- Sources of between-study heterogeneity and their independent contribution to CT prevalence assessed using meta-regression analyses (Table 2).

\section{RESULTS}

- 519 CT prevalence measures identified in MENA, of which 496 were eligible for analysis (Fig. 1).

- Estimates for the pooled mean CT prevalence (and 95\% confidence intervals) are found in Table 1.

- At-risk population, assay type, sample size, country and sex were identified as predictors of between-study heterogeneity in CT prevalence in Table 2.

Table 1. Findings of the meta-analyses of CT prevalence studies in MENA by at-risk population and CT ascertainment.

\begin{tabular}{|c|c|c|c|c|c|c|c|c|}
\hline \multirow[b]{2}{*}{ CT infection ascertainment } & \multirow{2}{*}{$\begin{array}{l}\text { Studies } \\
\mathrm{N} \\
\end{array}$} & \multicolumn{2}{|l|}{ Sample } & \multicolumn{3}{|c|}{ CT prevalence } & \multicolumn{2}{|c|}{ Heterogeneity measures } \\
\hline & & Tested & $\mathrm{CT}+\mathrm{ve}$ & Range (\%) & $\begin{array}{l}\begin{array}{l}\text { Pooled } \\
\text { mean (\%) }\end{array}\end{array}$ & $95 \% \mathrm{CI}$ & $\mathrm{I}^{\mathrm{P}^{* *}}(\% ; 95 \% \mathrm{Cl})$ & $\begin{array}{l}\begin{array}{l}\text { Prediction } \\
\text { interval }^{+}(95 \%)\end{array}\end{array}$ \\
\hline \multirow{2}{*}{\multicolumn{9}{|c|}{$\begin{array}{l}\text { General populations } \\
\text { Current genital infection }\end{array}$}} \\
\hline & & & & & & & & \\
\hline PCR (current infection) & 38 & 24299 & 671 & $0-12 \cdot 8$ & $3 \cdot 0$ & $2 \cdot 0-4 \cdot 1$ & $93 \cdot 8(92 \cdot 4-95 \cdot 0)$ & $0-11 \cdot 6$ \\
\hline Culture (current infection) & 6 & 5020 & 55 & $0-15 \cdot 0$ & 1.5 & $0 \cdot 1-4 \cdot 2$ & $86 \cdot 1(71 \cdot 8-93 \cdot 1)$ & $0-13 \cdot 6$ \\
\hline DFA (current infection) & 13 & 2052 & 66 & $0-12 \cdot 5$ & $2 \cdot 7$ & $1 \cdot 4-4 \cdot 2$ & $64 \cdot 0(34 \cdot 8-80 \cdot 1)$ & $0-8 \cdot 7$ \\
\hline \multirow{2}{*}{\multicolumn{9}{|c|}{ Anti-CT immunoglobulins }} \\
\hline & & & & & & & & \\
\hline IgG (lifetime exposure) & 28 & 5158 & 353 & $0-22 \cdot 2$ & $6 \cdot 0$ & $4 \cdot 1-8 \cdot 1$ & $83 \cdot 5(77 \cdot 2-88 \cdot 1)$ & $0 \cdot 04-18 \cdot 3$ \\
\hline IgM (recent infection) & 12 & 2718 & 72 & $0-14 \cdot 0$ & 1.8 & $0 \cdot 3-4 \cdot 2$ & $85 \cdot 8(77 \cdot 0-91 \cdot 3)$ & $0-13 \cdot 5$ \\
\hline $\begin{array}{l}\text { IgA (recent infection) } \\
\text { Nots secified }\end{array}$ & 3 & 317 & 8 & $0-40 \cdot 4$ & $6 \cdot 5$ & $0-29 \cdot 5$ & $93 \cdot 5$ (84:5-9-97-3) & $0-100$ \\
\hline $\begin{array}{l}\text { Not specified } \\
\text { Unclear }\end{array}$ & ${ }^{11}$ & 3300 & 187 & $0-19 \cdot 9$ & $6 \cdot 6$ & $4 \cdot 1-9 \cdot 6$ & $\begin{array}{l}87 \cdot 2(79 \cdot 1-92 \cdot 2) \\
\end{array}$ & \\
\hline \multirow{2}{*}{\multicolumn{9}{|c|}{ Populations at intermediate risk }} \\
\hline $\begin{array}{l}\text { Current genital infection } \\
\text { S }\end{array}$ & & & & & & & & \\
\hline PCR (current infection) & 12 & 2836 & 66 & $0-26 \cdot 1$ & $2 \cdot 3$ & $0 \cdot 8-4 \cdot 6$ & $88 \cdot 7(82 \cdot 1-92 \cdot 8)$ & $0-13 \cdot 7$ \\
\hline Overall (current infection) & 12 & 2836 & 66 & $0-26 \cdot 1$ & $2 \cdot 3$ & $0 \cdot 8-4 \cdot 6$ & $88 \cdot 7(82 \cdot 1-92 \cdot 8)$ & $0-13 \cdot 7$ \\
\hline Anti-CT immunoglobulins & & & & & & & & \\
\hline $\begin{array}{l}\text { IgG (lifetime exposure) } \\
\text { Not secified }\end{array}$ & 2 & $\begin{array}{l}1133 \\
308\end{array}$ & 31 & $1 \cdot 4-17 \cdot 4$ & $\begin{array}{l}7 \cdot 1 \\
4 \cdot 0\end{array}$ & $0-30 \cdot 0$ & $97 \cdot 0(92 \cdot 1-98 \cdot 8)$ & - \\
\hline $\begin{array}{l}\text { Not specified } \\
\text { Populations at high risk }\end{array}$ & 1 & 308 & 15 & & $4 \cdot 9$ & $2 \cdot 8-7 \cdot 9$ & & - \\
\hline $\begin{array}{l}\text { Populanons a ring risk } \\
\text { Current genital infection }\end{array}$ & & & & & & & & \\
\hline PCR (current infection) & 29 & 5701 & 455 & $0-72 \cdot 9$ & $5 \cdot 3$ & $2 \cdot 0-10 \cdot 0$ & $97 \cdot 6(97 \cdot 1-98 \cdot 1)$ & $0-38 \cdot 3$ \\
\hline Culture (current infection) & 1 & 116 & 8 & - & $6 \cdot 9$ & $3 \cdot 0-13 \cdot 1$ & क्ष & - \\
\hline DFA (current infection) & 1 & 30 & 9 & - & 30.0 & $14 \cdot 7-49 \cdot 4$ & - & - \\
\hline Current rectal infection & & & & & & & & \\
\hline PCR (current infection) & 7 & 1506 & 129 & $3 \cdot 6-18 \cdot 3$ & $7 \cdot 7$ & $4 \cdot 2-12 \cdot 0$ & $85 \cdot 2(71 \cdot 5-92 \cdot 3)$ & $0-24 \cdot 9$ \\
\hline Overall (current infection) & 31 & 7353 & 601 & $0-72 \cdot 9$ & $6 \cdot 3$ & $3 \cdot 4-10 \cdot 0$ & $96 \cdot 8(96 \cdot 2-97 \cdot 4)$ & $0-35 \cdot 3$ \\
\hline Anti-CT immunoglobulins & & & & & & & & \\
\hline $\operatorname{lgG}$ (lifetime exposure) & 4 & 472 & 364 & $19 \cdot 8-100$ & 80.9 & 43.8-100 & $98 \cdot 6(97 \cdot 7-99 \cdot 1)$ & $0-100$ \\
\hline $\begin{array}{l}\text { IgM (recent infection) } \\
\text { Not snecified }\end{array}$ & 1 & 154 & 45 & - & $29 \cdot 2$ & $\begin{array}{l}22 \cdot 2-37 \cdot 1 \\
10.0-56 \cdot 1\end{array}$ & - & - \\
\hline $\begin{array}{l}\text { Not specified } \\
\text { Infertility clinic attendees }\end{array}$ & 1 & 30 & 11 & - & $36 \cdot 7$ & $19 \cdot 9-56 \cdot 1$ & - & $\cdot$ \\
\hline Current genital infection & & & & & & & & \\
\hline PCR (current infection) & 26 & 4073 & 468 & $0-39 \cdot 4$ & 10.8 & $7 \cdot 7-14 \cdot 3$ & $90 \cdot 5(87 \cdot 3-92 \cdot 9)$ & $0 \cdot 2-32 \cdot 5$ \\
\hline Culture (current infection) & 8 & 1445 & 188 & $1 \cdot 1-65 \cdot 3$ & 12.7 & $4 \cdot 8-23 \cdot 6$ & $95 \cdot 9(93 \cdot 7-97 \cdot 3)$ & $0-59 \cdot 4$ \\
\hline DFA (current infection) & 13 & 932 & 131 & $4 \cdot 3-33 \cdot 0$ & 15.0 & $10 \cdot 5-20 \cdot 2$ & $74 \cdot 0(55 \cdot 0-85 \cdot 0)$ & $2 \cdot 1-35 \cdot 5$ \\
\hline Overall (current infection) & 47 & 6450 & 787 & $0-65 \cdot 3$ & $12 \cdot 2$ & $10 \cdot 0-15 \cdot 1$ & $90 \cdot 5(88 \cdot 3-92 \cdot 4)$ & $0 \cdot 3-35 \cdot 2$ \\
\hline 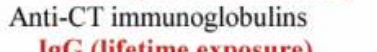 & & & & & & & & \\
\hline $\operatorname{lgG}$ (lifetime exposure) & 32 & 3774 & 752 & $0-53 \cdot 3$ & $21 \cdot 9$ & $16 \cdot 9-27 \cdot 4$ & $92 \cdot 9(91 \cdot 0-94 \cdot 4)$ & $1 \cdot 3-55 \cdot 9$ \\
\hline $\operatorname{lgM}$ (recent infection) & 14 & 3045 & 326 & $0-86 \cdot 7$ & $11 \cdot 5$ & $5 \cdot 5-19 \cdot 3$ & $97 \cdot 0(96 \cdot 0-97 \cdot 7)$ & $0-51 \cdot 1$ \\
\hline $\operatorname{IgA}$ (recent infection) & 7 & 2394 & 90 & $0-8.7$ & $2 \cdot 4$ & $0 \cdot 5-5 \cdot 5$ & $90 \cdot 1(82 \cdot 1-94 \cdot 5)$ & $0-16 \cdot 4$ \\
\hline Not specified & 5 & 847 & 178 & $5 \cdot 5 \cdot 32 \cdot 3$ & 18.0 & $8 \cdot 1-30 \cdot 8$ & 94-4 (89:8-9970) & $0-72 \cdot 2$ \\
\hline Unclear & 6 & 486 & 77 & $3 \cdot 3-33 \cdot 0$ & $14 \cdot 7$ & $6 \cdot 8-24 \cdot 6$ & $85 \cdot 2(69 \cdot 8-92 \cdot 8)$ & $0-53 \cdot 7$ \\
\hline $\begin{array}{l}\text { Women with miscarriage or ec } \\
\text { Current genital infection }\end{array}$ & regnancy & & & & & & & \\
\hline $\begin{array}{l}\text { PCR (current infection) } \\
\text { Pour }\end{array}$ & 3 & 256 & 36 & $1 \cdot 4-22 \cdot 9$ & $10 \cdot 8$ & $1 \cdot 4-26 \cdot 9$ & $90 \cdot 9(76 \cdot 4-96 \cdot 5)$ & $0-100$ \\
\hline Culture (current infection) & 2 & 112 & 7 & $5 \cdot 0-16 \cdot 7$ & $7 \cdot 1$ & $0-21 \cdot 8$ & $52 \cdot 1(0-88 \cdot 0)$ & \\
\hline DFA (current infection) & 3 & 434 & 76 & $7 \cdot 2-25 \cdot 5$ & $14 \cdot 4$ & $4 \cdot 9-27 \cdot 6$ & $90 \cdot 8(76 \cdot 1-96 \cdot 5)$ & $0-100$ \\
\hline Overall (current infection) & 8 & 802 & 119 & $1 \cdot 4-25 \cdot 5$ & $11 \cdot 5$ & $5 \cdot 6-19 \cdot 2$ & $87 \cdot 5(77 \cdot 6-93 \cdot 0)$ & $0-42 \cdot 5$ \\
\hline Anti-CT immunoglobulins & & & & & & & & \\
\hline IgG (lifetime exposure) & 7 & 512 & 83 & $6 \cdot 4-45 \cdot 0$ & $19 \cdot 8$ & $10 \cdot 4-31 \cdot 1$ & $85 \cdot 8(72 \cdot 7-92 \cdot 6)$ & $0-61 \cdot 0$ \\
\hline IgM (recent infection) & 6 & 376 & 83 & $0-38 \cdot 3$ & 18.0 & $9 \cdot 3-28 \cdot 6$ & $77 \cdot 4(49 \cdot 9-89 \cdot 8)$ & $0-56 \cdot 0$ \\
\hline Not specified & 2 & 149 & 13 & $0-14 \cdot 6$ & $4 \cdot 7$ & $0-27 \cdot 9$ & $93 \cdot 8(80 \cdot 0-98 \cdot 1)$ & $\cdot$ \\
\hline Unclear & 1 & 84 & 2 & & $2 \cdot 3$ & $0 \cdot 3-8 \cdot 3$ & & - \\
\hline 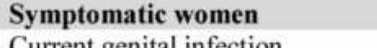 & & & & & & & & \\
\hline $\begin{array}{l}\text { Current genital infection } \\
\text { PCR (current iffection) }\end{array}$ & & & & & & & & \\
\hline $\begin{array}{l}\text { PCR (current infection) } \\
\text { Culture (usrent infection) }\end{array}$ & 48 & 14360 & $\begin{array}{l}1116 \\
831\end{array}$ & $\begin{array}{l}0-68 \cdot 0 \\
0 \cdot 7-89 \cdot 3\end{array}$ & $8 \cdot 7$ & $6 \cdot 1-11 \cdot 6$ & $\begin{array}{l}96 \cdot 9(96 \cdot 4-97 \cdot 3) \\
\end{array}$ & $0-35 \cdot 1$ \\
\hline $\begin{array}{l}\text { Culture (current infection) } \\
\text { DeA }\end{array}$ & 13 & 3085 & 831 & $0 \cdot 7-89 \cdot 3$ & $25 \cdot 7$ & $9 \cdot 4 \cdot 46 \cdot 6$ & $99 \cdot 3(99 \cdot 1-99 \cdot 4)$ & $0-98 \cdot 7$ \\
\hline $\begin{array}{l}\text { DFA (current infection) } \\
\text { Overall (current infection) }\end{array}$ & $\begin{array}{l}18 \\
79\end{array}$ & $\begin{array}{l}2046 \\
19491\end{array}$ & $\begin{array}{l}32963 \\
2293\end{array}$ & $\begin{array}{l}0-68 \cdot 0 \\
0-89 \cdot 3\end{array}-3$ & $\begin{array}{l}16 \cdot 6 \\
12 \cdot 7\end{array}$ & $\begin{array}{l}10 \cdot 5-23 \cdot 7 \\
9 \cdot 4-16 \cdot 5\end{array}$ & $\begin{array}{l}93 \cdot(90.5-95 \cdot 0) \\
98 \cdot(97 \cdot 9-98 \cdot 3)\end{array}$ & $\begin{array}{l}0-52.6 \\
0.546\end{array}$ \\
\hline Anti-CT immunoglobulins & & & & & & & & \\
\hline IgG (lifetime exposure) & 25 & 2607 & 657 & $0-86 \cdot 0$ & $21 \cdot 5$ & $14 \cdot 6-29 \cdot 2$ & $95 \cdot 1(93 \cdot 8-96 \cdot 2)$ & $0-65 \cdot 9$ \\
\hline IgM (recent infection) & 9 & 1042 & 160 & $0-86 \cdot 0$ & $13 \cdot 9$ & $1 \cdot 9-33 \cdot 6$ & $98 \cdot 2(97 \cdot 6-98 \cdot 7)$ & $0-91 \cdot 3$ \\
\hline IgA (recent infection) & 5 & 365 & 93 & $3 \cdot 7-59 \cdot 6$ & $24 \cdot 9$ & $6 \cdot 6-49 \cdot 6$ & $96 \cdot 0(93 \cdot 0-97 \cdot 7)$ & $0-100$ \\
\hline Not specified & 14 & 4672 & 1017 & $2 \cdot 6-46 \cdot 6$ & $17 \cdot 9$ & $9 \cdot 8-27 \cdot 9$ & $98 \cdot 4(98 \cdot 0-98 \cdot 7)$ & $0-65 \cdot 6$ \\
\hline Unclear & 8 & 1423 & 136 & $1 \cdot 7-30 \cdot 0$ & $8 \cdot 7$ & $5 \cdot 2-12 \cdot 8$ & $81 \cdot 9(65 \cdot 5-90 \cdot 5)$ & $0 \cdot 3-24 \cdot 9$ \\
\hline $\begin{array}{l}\text { Symptomatic men } \\
\text { Current genital infe }\end{array}$ & & & & & & & & \\
\hline $\begin{array}{l}\text { Current genital infection } \\
\text { PCR (current infection) }\end{array}$ & & 7160 & 726 & $4 \cdot 2-33 \cdot 3$ & & $8 \cdot 3-20 \cdot 6$ & & \\
\hline $\begin{array}{l}\text { PCR (current infection) } \\
\text { Culture (current tinfection) }\end{array}$ & ${ }_{5}^{14}$ & 4744 & 75 & $0 \cdot 4-19 \cdot 6$ & $8 \cdot 7$ & $1 \cdot 1-21 \cdot 7$ & $\begin{array}{l}97 \cdot 3 \cdot 3(96 \cdot 5 \cdot-98-08 \cdot 3) \\
97 \cdot 3)\end{array}$ & $\begin{array}{l}0-46.4 \\
0-72 \cdot 7\end{array}$ \\
\hline $\begin{array}{l}\text { Cuturece (current intection) } \\
\text { DFA (current infection) }\end{array}$ & 13 & 2288 & 335 & $4 \cdot 7 \cdot 52 \cdot 0$ & $29 \cdot 4$ & $16 \cdot 1-44 \cdot 6$ & $97 \cdot 0(95 \cdot 9-97 \cdot 7)$ & $0-88 \cdot 4$ \\
\hline Overall (current infection) & 32 & 14192 & 1136 & $0 \cdot 4-52 \cdot 0$ & $18 \cdot 4$ & $13 \cdot 3-24 \cdot 0$ & $98 \cdot 1(97 \cdot 8-98 \cdot 4)$ & $0-55 \cdot 5$ \\
\hline Anti-CT immunoglobulins & & & & & & & & \\
\hline $\operatorname{lgG}$ (lifetime exposure) & 8 & 831 & 164 & $5 \cdot 1-46 \cdot 0$ & $16 \cdot 9$ & $9 \cdot 4-25 \cdot 8$ & $89 \cdot 9(82 \cdot 6-94 \cdot 2)$ & $0-52 \cdot 8$ \\
\hline $\operatorname{lgM}$ (recent infection) & 3 & 330 & 24 & $2 \cdot 8-12 \cdot 2$ & $6 \cdot 0$ & $1 \cdot 4-13 \cdot 0$ & $78 \cdot 5(31 \cdot 0-93 \cdot 3)$ & $0-100$ \\
\hline $\begin{array}{l}\text { Not specified } \\
\text { Lnclar }\end{array}$ & 7 & 1952 & 719 & $\begin{array}{l}5 \cdot 7-49 \cdot 1 \\
1 \cdot 6 \cdot 7 \cdot 9\end{array}$ & $\begin{array}{l}21.5 \\
31.3\end{array}$ & $\begin{array}{l}8 \cdot 6-38 \cdot 0 \\
11.255 .9\end{array}$ & $\begin{array}{l}97 \cdot 9(96 \cdot 9-98 \cdot 5) \\
90 \cdot 0.08 \cdot-09 \cdot 3)\end{array}$ & $0-83 \cdot 0$ \\
\hline & & & & & & & & \\
\hline
\end{tabular}

Table 2. Meta-regression findings for the sources of between-study heterogeneity in CT prevalence in MENA

\begin{tabular}{|c|c|c|c|c|c|c|}
\hline \multirow[b]{2}{*}{ Predictors } & & \multirow{2}{*}{\begin{tabular}{|l|l|} 
Studies \\
Total N \\
\end{tabular}} & \multicolumn{2}{|c|}{ Univariable analyses } & \multicolumn{2}{|c|}{ Multivariable analysis } \\
\hline & & & $\mathrm{OR}(95 \% \mathrm{CI})$ & p-value* & AOR $(95 \% \mathrm{CI})$ & p-value" \\
\hline & General populations & 113 & $1 \cdot 00$ & & $1 \cdot 00$ & \\
\hline & Populat & 15 & $0 \cdot 76(0 \cdot 35-1 \cdot 66)$ & $0 \cdot 488$ & $2 \cdot 13(1.01-4 \cdot 48)$ & $0 \cdot 047$ \\
\hline & Popul: & 37 & $2 \cdot 50(1 \cdot 46-4 \cdot 29)$ & 0.001 & $8.08(4 \cdot 55-14 \cdot 34)$ & $<0 \cdot 0001$ \\
\hline \multirow{4}{*}{$\begin{array}{l}\text { At-risk } \\
\text { population }\end{array}$} & Infert & 111 & $3 \cdot 84(2 \cdot 62-5 \cdot 61)$ & $<0.0001$ & $3 \cdot 76(2 \cdot 64-5 \cdot 35)$ & $<0.0001$ \\
\hline & ectopic pregnancy & 24 & $4.01(2 \cdot 11-7 \cdot 61)$ & $<0.0001$ & $2.76(1.53-4.98)$ & 0.001 \\
\hline & Sympto & 140 & $3 \cdot 71(2 \cdot 59-5 \cdot 32)$ & $<0.0001$ & $3 \cdot 51(2 \cdot 51-4 \cdot 90)$ & $<0.0001$ \\
\hline & Symptomatic men & 56 & $5 \cdot 64(3 \cdot 54-8 \cdot 99)$ & $<0 \cdot 0001$ & $9 \cdot 65(5 \cdot 61-16 \cdot 61)$ & $<0.0001$ \\
\hline \multirow{8}{*}{ Assay type } & PCR & 170 & & & 1 . & \\
\hline & Cultur & 35 & $1 \cdot 94(1 \cdot 12-3 \cdot 36)$ & $0 \cdot 0$ & $1 \cdot 29(0 \cdot 77-2 \cdot 16)$ & 0.333 \\
\hline & DFA (current $\mathrm{i}$ & 61 & $2 \cdot 69(1 \cdot 73-4 \cdot 17)$ & $<0.0001$ & $1 \cdot 97(1 \cdot 28-3 \cdot 04)$ & $0 \cdot 002$ \\
\hline & Anti-C & & & & & \\
\hline & & $\begin{array}{l}106 \\
45\end{array}$ & & $<0.0001$ & $\begin{array}{l}2 \cdot 28(1 \cdot 60-3 \cdot 25) \\
0.94(0.60-48)\end{array}$ & $\begin{array}{l}<0.0001 \\
0.795\end{array}-1$ \\
\hline & $\begin{array}{l}\text { IgM (recent infection) } \\
\text { IgA (recent infection) }\end{array}$ & $\begin{array}{l}45 \\
15\end{array}$ & $\begin{array}{l}1.27(0.7-7-2.08) \\
1.02(0 \cdot 46-2 \cdot 25)\end{array}$ & $\begin{array}{l}0.343 \\
0.966\end{array}$ & $\begin{array}{l}0.94(0.60-1.48) \\
0.82(0 \cdot 41-1 \cdot 66)\end{array}$ & $\begin{array}{l}0.95 \\
0.584\end{array}$ \\
\hline & Not specified (IgG, IgM, or IgA) & 41 & $2 \cdot 55(1 \cdot 52-4 \cdot 26)$ & $<0.0001$ & $2 \cdot 05(1 \cdot 28-3 \cdot 28)$ & 0.003 \\
\hline & Unclear & 23 & $2 \cdot 10(1 \cdot 09-4 \cdot 04)$ & 0.027 & $1.44(0.79-2.62)$ & 0.231 \\
\hline
\end{tabular}

Sample size $\quad \begin{aligned}<200 \\ \geq 200\end{aligned}$

Year of
publication

Year of data

collection (median)
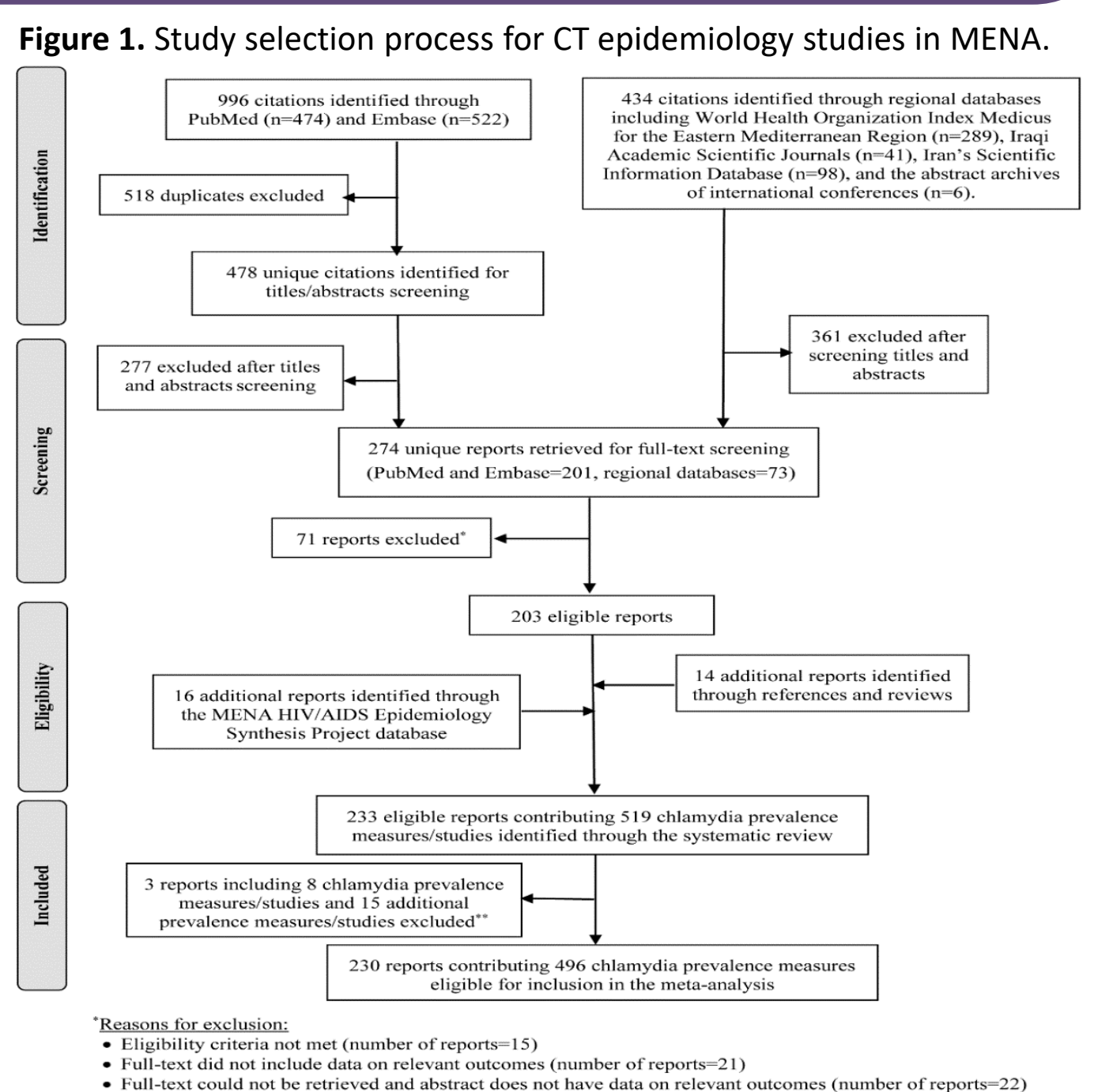

"Reasons for exclusion:
: Studies applying the same assay to different biological specimens (number of studies= 14 )
Studies conducted on subsamples (number of studies $=2$ )

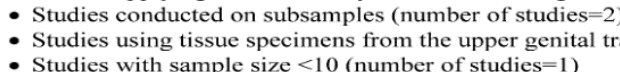

\begin{tabular}{|c|c|c|c|c|c|c|}
\hline \multirow{4}{*}{ Country } & Other MENA countries & 226 & $1 \cdot 00$ & \multicolumn{3}{|c|}{$1 \cdot 00$} \\
\hline & Egypt & 83 & $1 \cdot 61(1 \cdot 10-2 \cdot 36)$ & $0 \cdot 015$ & $0.92(0 \cdot 65-1 \cdot 32)$ & $0 \cdot 660$ \\
\hline & & 145 & $0.83(0.60-1.14)$ & 0.243 & $0.85(0.64-1.12)$ & 0.243 \\
\hline & Pakistan & 42 & $0.28(0.17-0.47)$ & $<0.0001$ & $0.29(0.17-0.49)$ & $<0.0001$ \\
\hline Sex & Men & 126 & 1.00 & & $1 \cdot 00$ & \\
\hline
\end{tabular}

"*Predictors with $p$-value $\leq 0.1$ were eligible for inclusion in the multivariable analysis.

"Predictors with $\mathrm{p}$-value $<0.05$ were retained in the final multivariable model.

Adjusted $R^{2}$ in the final multivariable

AOR=Adjusted odds ratio. Cl=Confidence interval. OR=Odds ratio.

CONCLUSIONS

- CT prevalence in MENA's general populations is comparable to

global levels.

- The high CT prevalence in infertility clinic attendees and in women with miscarriage or ectopic pregnancy may suggest considerable

CT disease burden with important socio-cultural implications.

- CT transmission appears to be driven by pockets of high sexual risk behavior.

- Targeted and culturally-sensitive sexual health programs including screening services for at-risk populations are warranted.

FUNDING ACKNOWLEDGMENT

This publication was made possible by NPRP grant number 9-040-3-008 from the Qatar National Research Fund (a member 